A N N A L E S Annales de Bretagne et des Pays de l'Ouest

\title{
Codicille aux testaments angevins au XVIIIe siècle : les charités
}

\section{Philippe Haudrère}

\section{(2) OpenEdition \\ 1 Journals}

Édition électronique

URL : http://journals.openedition.org/abpo/2232

DOI : $10.4000 / a b p o .2232$

ISBN : 978-2-7535-1841-4

ISSN : 2108-6443

Éditeur

Presses universitaires de Rennes

Édition imprimée

Date de publication : 30 décembre 2011

Pagination : $79-89$

ISBN : 978-2-7535-1839-1

ISSN : 0399-0826

\section{Référence électronique}

Philippe Haudrère, "Codicille aux testaments angevins au XVIIle siècle : les charités », Annales de Bretagne et des Pays de l'Ouest [En ligne], 118-4 | 2011, mis en ligne le 30 décembre 2013, consulté le 30 novembre 2020. URL : http://journals.openedition.org/abpo/2232 ; DOI : https://doi.org/10.4000/ abpo.2232 


\title{
Codicille aux testaments angevins au XVIII ${ }^{\mathrm{e}}$ siècle : les charités
}

\author{
Philippe HAUDRÈRE \\ professeur émérite à l'université d'Angers
}

Dans un précédent numéro des Annales de Bretagne et des Pays de l'Ouest, en 2009, j'ai essayé de présenter les résultats d'une enquête sur la pratique testamentaire en Anjou, réalisée par vingt-sept étudiants d'histoire de l'Université d'Angers, et portant sur plus de 9000 testaments, dont les trois quarts dans des paroisses rurales ${ }^{1}$. Cette enquête, conduite avec la même méthode que celle de M. Vovelle en Provence et de P. Chaunu à Paris ${ }^{2}$, avait pour objet de chercher les indices de l'évolution de la pratique catholique en Anjou.

Il est apparu qu'un huitième des habitants des campagnes et un sixième de ceux des villes faisaient un testament contenant des dispositions pieuses. Après un point culminant, situé entre 1710 et 1740 , commence un lent mouvement de déclin; celui-ci s'accélère après 1760 , et c'est une véritable débâcle à partir de 1780, sans qu'il se produise toutefois une disparition totale. Pour mesurer l'évolution de la piété testamentaire, quatre indices ont été retenus. Le premier est le choix de l'exécuteur testamentaire; sur l'ensemble du siècle, les clercs (44\%) l'emportent sur les laïcs (41 \%), cependant à partir de la décennie 1760-1770, la proportion s'inverse au profit des laïcs (44\%) et la place de ceux-ci ne cesse de croitre. Le second critère est la " recommandation " de l'âme à Dieu; c'est encore une pratique en diminution, avec $96 \%$ des testaments dans la décennie 1700 à 1710, $76 \%$ en 1750-1760, 51 \% en 1780-1790. Le troisième critère est le recours à des intercesseurs célestes pour obtenir l'entrée de l'âme en Paradis : une fois encore, c'est une dévotion en voie de disparition, avec $60 \%$ de demandes

1. Annales de Bretagne et des Pays de l'Ouest, t. 116, 2009 (2), p. 105-119.

2. Vovelle, Michel, Piété baroque et déchristianisation en Provence au XVIII siècle : les attitudes devant la mort d'après les clauses des testaments, Paris, 1973; réédition partielle avec une étude sur vingt-cinq années d'historiographie, Paris, Comité des travaux historiques et scientifiques, 1997; Chaunu, Pierre, La Mort à Paris, XVI ${ }^{e}$, XVII et XVIII $T^{e}$ siècles, Paris, 1978. Sur le don en général, voir l'introduction de MARAIS, Jean-Luc, Histoire du don en France de 1800 à 1939, Rennes, 1999, p. 7-23. 
en $1700-1710$, et $25 \%$ entre 1780 et 1789 . Le dernier indice retenu est l'évolution de la demande de messes; celle-ci est forte entre 1710 et 1740 , avec une moyenne de 72 messes par testament; de 1740 à 1770 , il y a un effritement progressif, et la moyenne passe à 54 messes; entre 1770 et 1790 , le mouvement se poursuit, avec une moyenne de 32 messes par testament.

Après avoir lu cet article, plusieurs de mes correspondants ont regretté l'absence d'une étude sur l'évolution des dons charitables, alors que le relevé de ceux-ci figurait dans le programme de l'enquête conduite par les étudiants. La présente note cherche à combler cette lacune et me permet de remercier à nouveau les vingt-sept étudiants ${ }^{3}$ qui ont accepté de travailler à ce dépouillement d'archives. Sans leur activité assidue, rien n'aurait pu être fait.

Il apparaît tout d'abord qu'un quart des testaments (2353 sur un total de 9085) contiennent des dons et legs charitables.

C'est une pratique fortement affirmée par le Christ, en particulier dans l'Évangile de Saint Matthieu (chap. 25, v. 34 à 40) à l'occasion d'une description du jugement dernier : "Venez les bénis de mon Père, recevez en partage le Royaume qui a été préparé pour vous... Car j'ai eu faim et vous m'avez donné à manger... [j'ai été] nu et vous m'avez vêtu; malade, et vous m'avez visité; en prison, et vous êtes venu à moi. " Alors les justes lui répondront : "Seigneur, quand nous est-il arrivé de te voir affamé et de te nourrir... nu et de te vêtir? " Et il leur dira : "En vérité, je vous le déclare, chaque fois que vous l'avez fait à l'un de ces plus petits, qui sont mes frères, c'est à moi que vous l'avez fait! "Ce thème est abondamment développé dans les sermons des grands prédicateurs lorsqu'ils s'adressent aux riches : "Dans l'Église de Jésus-Christ, vous êtes seulement les serviteurs des pauvres ", énonce Bossuet ${ }^{4}$; "le riche n'est pas riche pour lui-même, mais pour les pauvres " assure Bourdaloue ${ }^{5}$. Et le père Crasset, dans son livre La douce et sainte mort, dont le succès de librairie est grand, écrit : "Un simple acte d'amour envers Dieu peut effacer les péchés d'une vie entière ${ }^{6}$. "

\section{" J'ai eu faim et vous m'avez donné à manger... "}

La nature des dons répond aux préceptes de l'Évangile. En premier lieu vient le pain ( $46 \%$ des donateurs) sous forme de "bled ", soit froment, soit seigle, soit le plus souvent " méteil ", c'est-à-dire moitié froment et moitié seigle. Les quantités sont exprimées en boisseaux (de 12,8 litres) et setiers (de 1,52 hectolitres, soit 12 boisseaux), à raison de 500 litres en moyenne par testament, ce qui est beaucoup. Dans la moitié des actes la distribution est prévue en grains, dans un quart en farine ou "mouture ", et le reste en pain. Les dons en "bled " sont toujours majoritaires dans les régions

3. Dont les noms sont cités dans le précédent article.

4. Sur l'éminente dignité des pauvres, Euvres complètes, Paris, t. 3, p. 189.

5. Sermon sur l'aumône, Euvres complètes, Besançon, 1882, t. 4, p. 75.

6. Paris, 1698, p. 283. 
rurales; ils rivalisent avec les dons en argent dans les villes de Saumur (34\% en moyenne pour chaque nature de don) et Angers (33\% en grains et $34 \%$ en argent).

Dans l'ensemble de la province les dons en argent occupent la seconde place. Ils sont mentionnés dans $23 \%$ des testaments contenant des clauses charitables et la moyenne est de 110 livres tournois par acte.

Puis viennent les dons de vêtements (13\% des testaments), dont la valeur et la composition est en relation avec la fortune et le statut social du testateur ou de la testatrice. Ainsi la veuve d'un lieutenant général criminel de la sénéchaussée de Beaufort-en-Vallée déclare-t-elle " vouloir ordonner et entendre que pendant les vingt années qui suivront immédiatement son décès, il soit, le 15 octobre de chacune des dites vingt années, réparti et distribué par égale portion, à six jeunes garçons pauvres de cette paroisse et à six jeunes filles pauvres, aussi de cette paroisse, la quantité de quarante aunes de grosses et bonnes étoffes " ". La veuve d'un notaire de Mouliherne fait habiller sept enfants de 10 à 12 ans $^{8}$. Une fille majeure, domestique, résidant à Angers, donne à chacun de cinq pauvres de sa connaissance « deux chemises, deux paires de bas, une jupe, deux coiffes de jour, un tablier et deux mouchoirs, soit d'indienne ou de coton, chaque paquet d'effet valant vingt livres ${ }^{9}$ ". Une veuve de Rochefort-sur-Loire ordonne " que soit donné aux pauvres trois de mes moindres habillements complets ", et une fille majeure de Vernoil-le-Fourier, près de Baugé, ses " hardes, une douzaine de coiffures et une douzaine de mouchoirs ${ }^{10} "$.

Puis, en quatrième position avec $9 \%$, viennent les dons aux hôpitaux. Ceux-ci sont assez nombreux en Anjou ${ }^{11}$ et il y a même en 1704 la création d'une institution nouvelle à Saumur par Jeanne Delanoue, les filles de la Providence, mentionnées de plus en plus fréquemment au cours du $\mathrm{XVIII}^{\mathrm{e}}$ siècle dans les testaments ${ }^{12}$. La moyenne est de 480 livres tournois par donateur. Il y a aussi quelques dons pour l'achat de " remèdes ", ainsi

7. Marie Magdeleine Hauchère, 13 juin 1788, Arch. dép. de Maine-et-Loire, $5^{\mathrm{E} 54 / 982 .}$

8. Jeanne Gaultier, 6 juin 1763, Arch. dép. de Maine-et-Loire, $5^{\mathrm{E}} 77 / 64$.

9. Marie Chatelain, 26 août 1788, Arch. dép. de Maine-et-Loire, $5^{\mathrm{E}} 1 / 1131$.

10. Jeanne Allain, 13 décembre 1717, Arch. dép. de Maine-et-Loire, $5^{\mathrm{E} 39 / 15 .}$

11. Selon Muriel Jeorger ("La structure hospitalière de la France sous l'Ancien Régime ", Annales ESC, 1977, 5, p. 1051), la province se situe au treizième rang des départements français au début de la Révolution avec un indice des lits d'hôpitaux de 0,84 pour mille habitants, alors que le taux national est de 0,75 .

12. La mère des pauvres, Jeanne Delanoue, 1666-1730. Texte du manuscrit de Marie Laigle, Saint-Hilaire-Saint-Florent, 1968. Relevé des dons avec référence aux Arch. dép. de Maineet-Loire :

\begin{tabular}{|c|c|c|c|c|c|c|c|c|c|c|c|}
\hline & valeur & référence & & valeur & référence & & valeur & référence & & valeur & référence \\
\hline 1722 & 800 & $5 \mathrm{E} 43 / 9$ & 1774 & 100 & $5 \mathrm{E} 43 / 29$ & 1780 & 24 & $5 \mathrm{E} 43 / 434$ & 1789 & 500 & $5 \mathrm{E} 43 / 39$ \\
\hline 1736 & 80 & $5 \mathrm{E} 69 / 630$ & 1775 & 50 & $5 \mathrm{E} 43 / 25$ & 1784 & 832 & $5 \mathrm{E} 43 / 33$ & 1790 & 600 & $5 \mathrm{E} 69 / 669$ \\
\hline 1765 & 50 & $5 \mathrm{E} 69 / 467$ & 1776 & 100 & $5 \mathrm{E} 43 / 36$ & 1785 & 48 & $5 \mathrm{E} 43 / 38$ & & & \\
\hline 1772 & 100 & $5 \mathrm{E} 43 / 29$ & 1777 & 600 & $5 \mathrm{E} 43 / 433$ & 1788 & 90 & $5 \mathrm{E} 43 / 42$ & & & \\
\hline
\end{tabular}

Localisation des fonds : Archives départementales de Maine-et-Loire (Valeur en livres tournois) 
35 livres à Rochefort-sur-Loire en $1734^{13}$, un capital de 624 livres produisant une rente de 31 livres au Louroux-Béconnais en $1773^{14}, 50$ livres à Chateauneuf-sur-Sarthe en $1775^{15}$.

Les fabriques des paroisses reçoivent $9 \%$ des dons ordonnés dans les testaments, à égalité avec les hôpitaux. Cependant les affectations sont différentes puisque les fabriques doivent d'abord pourvoir "à l'entretien de l'église paroissiale, tant pour les réparations que pour la célébration du service " (A. Furetière, Dictionnaire universel, La Haye, 1690, t. 2) et ensuite seulement venir au secours des pauvres, à la diligence du procureur élu. Les trois quarts des dons sont affectés à la première dépense. En voici quelques exemples : le curé de Notre-Dame de Cholet donne 3000 livres à la fabrique "pour l'augmentation et la reconstruction de l'église ${ }^{16}$ "; le curé de la paroisse de Faye, proche de Saumur, écrit : " Je donne à la fabrique de ma paroisse un encensoir, trois chapes, deux dalmatiques, une chasuble, à remettre entre les mains du procureur ${ }^{17}$ "; une fille majeure de Candé affecte " la somme de 30 livres, qui sera employée à acheter une niche dorée, où reposera le Très Saint Sacrement ${ }^{18}$ "; un domestique de Durtal donne une belle tasse d'argent pour en faire un ciboire, "pour porter le Saint-Sacrement aux pauvres " de la paroisse de Saint-Léonard ${ }^{19}$. Ainsi le service des pauvres n'est-il pas loin. De même, un vicaire de la paroisse de Brain-sur-l'Authion donne-t-il la somme de 1600 livres à la fabrique, dont 1000 livres pour construire un nouveau maître-hôtel pour l'église et 600 pour l'achat d'un beau tabernacle, en précisant que si le conseil de la fabrique refuse le don la somme sera remise à l'hôpital général d'Angers pour les pauvres ${ }^{20}$ : Charles Briçonnet, baron de Lasse, lègue 300 livres à la fabrique de cette paroisse, située près de Saumur, en laissant la liberté d'affectation au procureur, mais en mettant les pauvres parmi les destinataires ${ }^{21}$.

Quelques testateurs créent des écoles pour l'éducation des enfants pauvres. Ainsi en 1700, Françoise Henry, veuve de Daniel de Landry, écuyer, fonde-t-elle une rente de 100 livres par an pour la subsistance d'une maîtresse d'école dans la paroisse de Bourg. Celle-ci devra enseigner " la lecture, les mystères de la religion et la manière de bien vivre ${ }^{22}$ ". En 1703, Guillaume de Bautru, comte de Serrant, créé une rente annuelle de 150 livres, " pour la nourriture et l'entretien de deux filles de la charité qui feront l'école aux pauvres de la paroisse ${ }^{23}$ " de Saint-Georges-sur-

13. Marie Huet, 16 janvier 1734, Arch. dép. de Maine-et-Loire, $5^{\mathrm{E}} 52 / 55$.

14. Bibliothèque municipale d'Angers, Ms. 1842.

15. Marie Bâle, 3 juin $1775,5^{\mathrm{E}} 80 / 222$.

16. Joseph Lefèvre, 19 février 1774 , Arch. dép. de Maine-et-Loire, $5^{\mathrm{E}} 11 / 86$.

17. Joseph Corbin, 22 juillet 1747, Arch. dép. de Maine-et-Loire, $5^{\mathrm{E}} 69 / 449$.

18. Marie Gaudin, 8 février $1740,5^{\mathrm{E}} 72 /$ non coté.

19. André Montaugé, 10 décembre 1705, Arch. dép. de Maine-et-Loire, $5^{\mathrm{E}} 86 / 110$.

20. Jacques Morin, 14 octobre 1722, Arch. dép. de Maine-et-Loire, $5^{\mathrm{E}} 15 / 12$.

21. 18 janvier 1759, Arch. dép. de Maine-et-Loire, $5^{\mathrm{E}} 77 / 63$.

22. Arch. dép. de Maine-et-Loire, $5^{\mathrm{E}} 1 / 874$.

23. Arch. dép. de Maine-et-Loire, $5^{\mathrm{E}} 1 / 953$. 
Loire. Et en 1757, encore, Marguerite Renée de Bellesort donne la somme de 2000 livres " pour aider à fonder l'école de charité des filles de cette paroisse $^{24}$ ", de Rochefort-sur-Loire.

Les pauvres sont donc les principaux bénéficiaires des charités testamentaires. Des legs sont aussi remis aux domestiques : la moitié de ceux des nobles d'Angers et le dixième de ceux des marchands sont mentionnés dans les testaments de leurs maîtres. 38 \% de ces actes prévoient une "récompense " en argent, dont le montant moyen est de 150 livres; 31 \% le versement des gages de "l'année courante au décès "; $19 \%$ le don des meubles de leur logement, ainsi le " lit garni ", leurs vêtements et leur linge; 12 \% le paiement d'une rente viagère, dont le montant annuel se monte de 10 à 400 livres. Ce dernier legs est une faveur particulière, destinée à récompenser des services assidus. Ainsi Marie-Catherine Baudry, veuve de Nicolas Baudard de Saint-James, trésorier général de la Marine, donne-t-elle 100 livres de rente viagère annuelle à sa femme de chambre, et 150 livres, " payé en une fois ", à ses autres domestiques ${ }^{25}$; de même, Perrine Lacoudre, veuve d'un marchand, donne à sa "servante domestique " 30 livres de rente viagère, "payable chacun an " sur les revenus de ses biens immeubles ${ }^{26}$.

Quelques testateurs - un petit nombre - se préoccupent de réparer les "torts faits ". Ainsi Marie Drouault, veuve d'un marchand de SaintDenis-d'Anjou, " prie ceux qu'elle aurait offensé de lui pardonner comme elle pardonne de bon cœur à tous ceux qui l'ont offensé. Veut et ordonne ladite testatrice ses dettes être payées et que les torts qui se trouveraient être faits soient entièrement réparés par son exécuteur testamentaire ${ }^{27}$ "; Jeanne Trochon, veuve d'un notaire de Rochefort-sur-Loire ajoute 56 livres à un don de grain, " pour la tranquillité de sa conscience, pour pareillement le repos de son âme, pour réparation de quelques injustices qu'elle pourrait avoir fait en plusieurs et différents temps et à différentes personnes, qu'elle craint que son âme en demeura souillée ${ }^{28}$ ".

\section{Donateurs et bénéficiaires}

Qui donne? En dépit d'un nombre élevé d'indéterminés (41\%), il est possible d'apporter quelques précisions. Ce sont d'abord les plus riches, c'est-à-dire tous les clercs dont les testaments ont été relevés dans l'enquête des 9000 actes, la moitié des nobles, 14 \% des marchands et des représentants des professions " libérales "; $6 \%$ des "bourgeois " et des " officiers ". Puis viennent les maîtres de métier (1\%); les laboureurs, fermiers, métayers et vignerons (1,5\%); les compagnons, domestiques,

24. Arch. dép. de Maine-et-Loire, $5^{\mathrm{E}} 52 / 69$.

25. 30 décembre 1776, Arch. dép. de Maine-et-Loire, $5^{\mathrm{E} 90 / 614 .}$

26. 2 avril 1743, Arch. dép. de Maine-et-Loire, $5^{\mathrm{E}} 1 / 1044$.

27. 2 avril 1738, Arch. dép. de Maine-et-Loire, $5^{\mathrm{E}} 80 / 9$.

28. 15 janvier 1776, Arch. dép. de Maine-et-Loire, $5^{\mathrm{E}} 68 / 7$. 
journaliers et bêcheurs (0,5\%), les filles majeures (1,5\%). Il faut observer à ce propos que les dons en argent ne sont pas uniquement le fait des plus riches; certains testateurs, que l'on pourrait facilement qualifier de pauvres, lèguent des sommes importantes, ainsi Maurille Jeansimon, garçon sergé de Chalonnes-sur-Loire, un capital de 600 livres pour le soulagement des pauvres ${ }^{29}$; Marie Boumier, femme de journalier, 50 livres à la " maison de charité " de la fabrique de la paroisse de Rochefort-sur-Loire ${ }^{30}$; Jacques Daviaud, "serviteur domestique ", donne 25 livres aux pauvres de sa paroisse de Saint-Pierre de Cholet $^{31}$.

Le motif du don est clairement précisé : il s'agit d'engager les pauvres " à prier Dieu pour le repos de l'âme " du testateur. Parfois la demande se fait insistante : ainsi un bourgeois de Brissac remet-il 200 livres " entre les mains de Monsieur le Curé, le priant de faire exhorter les pauvres qui participeront à cette charité de prier pour le repos de son âme ${ }^{32}$ "; une fille majeure de la paroisse de la Trinité d'Angers donne 400 livres à des pauvres que son exécutrice testamentaire " choisira à sa volonté pour les obliger à prier Dieu pour le repos de son âme ${ }^{33}$ ". Quelquefois la demande devient émouvante, comme dans le testament olographe d'un noble qui fait un don aux pauvres "pour [leur faire] implorer miséricorde pour moi ${ }^{34}$ ".

La distribution des legs est en relation étroite avec la messe de sépulture et la messe de huitaine ou d'autres messes anniversaires. Dans la moitié des testaments le moment de la remise est précisé, à l'instar d'une paroissienne de Brissac : c'est " à l'issue des services où elle désire que les pauvres assistent, à l'effet de quoi elle prie le sieur curé de vouloir bien les inviter ${ }^{35}$ "; ou ce testateur de Rochefort-sur-Loire : "Que le jour de l'inhumation et le jour du sème [messe de huitaine] soit donné aux pauvres nécessiteux de la paroisse quatorze boisseaux de farine bled seigle, savoir la moitié le jour de la sépulture et l'autre le jour du sème ${ }^{36}$. "Un riche marchand organise scrupuleusement la distribution de ses dons en argent, à savoir : " 1000 livres aux pauvres de la paroisse de Saint-Pierre, à l'issue d'une messe dite à son intention; 200 livres aux pauvres de Notre-Dame, suivant le même ordre ". Les pauvres de La Séguinière et de Saint-Léger, recevront, pour chacune de ces paroisses toutes proches, la somme de 300 livres « en présence du procureur de la paroisse, à condition de faire dire une messe à son intention ${ }^{37}$ ". Une pratique analogue est parfois observée pour les dons de vêtements, ainsi une testatrice de Saint-Denis-d'Anjou demande-t-elle " que quatre fillettes de 4 à

29. 2 mai 1782, Arch. dép. de Maine-et-Loire, $5^{\mathrm{E}} 53 / 66$.

30. 22 mai 1783, Arch. dép. de Maine-et-Loire, $5^{\mathrm{E}} 53 / 67$.

31. 18 avril 1740, Arch. dép. de Maine-et-Loire, $5^{\mathrm{E}} 11 / 69$.

32. Gabriel Sénil, 2 juin 1740, Arch. dép. de Maine-et-Loire, $5^{\mathrm{E} 33 / 240 .}$

33. Catherine Guilbault, 16 décembre 1715, Arch. dép. de Maine-et-Loire, $5^{\mathrm{E}} 1 / 685$.

34. Henri Leroux de Noyen, Vernoil-le-Fourrier, 20 juillet 1757, Arch. dép. de Maine-etLoire, $5^{\mathrm{E}} 39 / 29$.

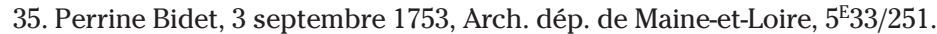

36. René Besnard, 15 mars 1713, Arch. dép. de Maine-et-Loire, $5^{\mathrm{E}} 52 / 39$.

37. Jacques Tellier, 27 décembre 1771, Arch. dép. de Maine-et-Loire, $5^{\mathrm{E}}$ 11/84. 
5 ans soient habillées selon sa volonté et se rendent à sa messe de sépulture en portant chacune un cierge ${ }^{38}$ ». Il en est de même encore dans les dons aux hôpitaux, à l'exemple du testament d'un notaire de Candé :

" comme le dit sieur a formé depuis longtemps le dessein de contribuer à l'augmentation de l'hôpital Saint-Joseph de cette ville, et pour participer aux prières des pauvres du dit hôpital et des dames hospitalières qui sont chargées de l'administration d'icelui, veut et entend le dit testateur qu'il soit donné un lit garni avec six draps et mille livres... et afin qu'on puisse s'en souvenir et en avoir mémoire du testateur, priant et suppliant ces dites dames hospitalières de faire souvenir les pauvres du dit hôpital du dit don et les engager d'avoir mémoire du testateur dans leurs prières et pour ce faire sera mis dans la chapelle du dit hôpital une plaque de cuivre qui contiendra en abrégé la présente donation ${ }^{39} "$.

Les dons à la fabrique relèvent parfois du même état d'esprit, ainsi une marchande de Rochefort-sur-Loire donne-t-elle 300 livres pour l'achat d'un ciboire ainsi que d'un "vaisseau " d'argent destiné à conserver les saintes huiles et demande que son nom " soit gravé sur la patte du Saint-Ciboire et sur le vaisseau des Saintes huiles où sera marqué qu'elle les a donnés... et que ce même don soit porté sur la croix mise au bout de sa fosse $\mathrm{e}^{40}$ ".

La difficulté rencontrée par les testateurs est de parvenir à distinguer ceux qui sont réellement dans le besoin. 57 \% des actes mentionnent les " pauvres " sans autre précision, et le choix appartient alors conjointement au curé, au procureur de la fabrique et à l'exécuteur testamentaire. Ainsi Thomas Halbert, de Brissac, précise-t-il dans son testament olographe : "Par pauvres, j'entends ceux qui ne vivent pas trop de leurs aises au dire des principaux de la paroisse qui les connaissent "; Jacquine Ragueau de Rochefort-sur-Loire, déjà citée, ordonne une distribution de " bled seigle " aux pauvres par son exécutrice testamentaire, " conjointement avec monsieur le curé "; Jacques Tellier, marchand de Cholet, aussi déjà cité, ordonne une distribution de 300 livres aux pauvres de deux paroisses de la proche banlieue, "en présence du procureur syndic de la paroisse".

Pour $13 \%$ des testateurs, les dons doivent être remis aux "nécessiteux ". Ainsi Claude Tavernier de Boullongne, receveur général des fermes du roi à Angers, lègue-t-il 1500 livres " aux pauvres les plus nécessiteux ${ }^{41}$ "; Françoise Beurois, de Beaufort-en-Vallée, "veut et ordonne qu'il soit, aussitôt son décès arrivé, distribué par son exécuteur testamentaire aux pauvres les plus nécessiteux de la dite paroisse ${ }^{42} \ldots$ "; Michel Lefébure, de Champigné, fait un don à l'hôpital Saint-Jean d'Angers pour « servir aux plus urgents nécessiteux des pauvres ${ }^{43}$ ".

38. Marie Salmon, 27 janvier 1729, Arch. dép. de Maine-et-Loire, $5^{\mathrm{E}} 80 / 6$.

39. Pierre Thébault, 22 août 1724, Arch. dép. de Maine-et-Loire, $5^{\mathrm{E}} 72 / 5$.

40. Jacquine Ragueau, 6 octobre 1716, Arch. dép. de Maine-et-Loire, $5^{\mathrm{E}} 52 / 13$.

41. 8 janvier 1779, Arch. dép. de Maine-et-Loire, $5^{\mathrm{E}} 7 / 665$.

42. 28 décembre 1763, Arch. dép. de Maine-et-Loire, $5^{5} 54 / 557$.

43. 18 septembre 1786, Arch. dép. de Maine-et-Loire, $5^{\mathrm{E}} 80 / 357$. 
$4 \%$ des legs sont pour des pauvres " honteux ", c'est-à-dire ceux qui ne mendient pas et qu'il faut secourir discrètement à domicile. André Bérard, de Saumur, ordonne de "donner aux pauvres honteux de la paroisse de Saint-Pierre la somme de cinquante livres ${ }^{44}$ ", et Gabriel Sénil, de Brissac, demande de faire " délivrer aux pauvres honteux de la paroisse, incontinent après son décès, deux cents livres, laquelle [somme] sera remise entre les mains de Monsieur le Curé ${ }^{45}$ ".

$3 \%$ des testateurs se préoccupent du sort des prisonniers : "J'étais en prison et vous êtes venu à moi ". Jean Garnier, de Saumur, lègue "Cent livres aux prisonniers des prisons royales et pareille somme aux prisonniers de la tour Grenetière [dans laquelle sont enfermés les contrebandiers de la gabelle] ${ }^{46}$ ". Louis Dupré, de Cherré, demande : " qu'il soit délivré la somme de dix livres aux pauvres prisonniers de Château-Gontier ${ }^{47}$ ", tandis que Marie Errand, de Beaufort-en-Vallée, lègue " 4 livres aux personnes chargées des pauvres pour prendre soin des pauvres prisonniers ${ }^{48}$ ".

Quelques testaments sont plus précis encore sur les pauvres auxquels il faut remettre les dons. Françoise Guyard, de Pouancé, prévoit " une fourniture de toile d'étoupe en réparon pour habiller des petits enfants orphelins $^{49}$ "; Michel Chupin, de Cholet, donne 500 livres, " pour assurer la subsistance des femmes pauvres en couches et celle de leurs enfants ${ }^{50}$ "; Perrine Bidet, "donne et lègue le plus tost son décès que faire se pourra aux pauvres incurables de la ville d'Angers la somme de cinq cents livres une fois payée, aux pauvres renfermés de la dite ville d'Angers la somme de deux cents livres aussi une fois payée ${ }^{51}$ ". Enfin Bernard Laumonnier, de Cherré, demande " qu'il soit donné à quatre des plus pauvres chacun 40 sols pour acheter des pelles à beicher et autres ferements propres à leur travail ${ }^{52}$ ".

Dans $14 \%$ des testaments les pauvres bénéficiaires sont "nommés et désignés ", ainsi Marguerite Lesnée, donne-t-elle 300 livres au pauvre Antoine Mesnard, "âgé de 40 ans, très infirme et en démence d'esprit depuis plusieurs années ${ }^{53}$ "; Jacques Ribault, de Rochefort-sur-Loire, précise que 6 setiers "seront pris et levés par préférence sur les quarante setiers de blé seigle ordonnés pour les pauvres nécessiteux de cette paroisse, regardant en chef ladite Anne Gouzil, sa servante, comme la première pauvre ${ }^{54}$ "; Marie Chatelain, fille majeure et domestique sur la paroisse de la Trinité

44. 15 janvier 1753, Arch. dép. de Maine-et-Loire, $5^{\mathrm{E}} 69 / 455$.

45. 2 juin 1740, Arch. dép. de Maine-et-Loire, $5^{\mathrm{E}} 33 / 244$.

46. 4 février 1741, Arch. dép. de Maine-et-Loire, $5^{\mathrm{E}} 69 / 635$.

47. 11 juillet 1732, Arch. dép. de Maine-et-Loire, $5^{\mathrm{E}} 80 / 375$.

48. 10 mars 1756, Arch. dép. de Maine-et-Loire, $5^{\mathrm{E} 54 / 562 .}$

49. 11 août 1721, Arch. dép. de Maine-et-Loire, $5^{\mathrm{E}} 20 / 176$.

50. 16 avril 1774, Arch. dép. de Maine-et-Loire, $5^{\mathrm{E}} 11 / 86$.

51. 3 septembre 1735, Arch. dép. de Maine-et-Loire, $5^{\mathrm{E}} 33 / 521$.

52. 23 octobre 1727, Arch. dép. de Maine-et-Loire, $5^{\mathrm{E}} 80 / 373$.

53. 11 mai 1775, Arch. dép. de Maine-et-Loire, $5^{\mathrm{E}} 11 / 87$.

54. 17 septembre 1717, Arch. dép. de Maine-et-Loire, 552/40. 
d'Angers, fait un même don de vêtements identiques à cinq personnes choisies, savoir : "La femme de Dubreuil, tessier, demeurante sur le Tertre Saint-Laurent, vis-à-vis le cimetière des pauvres; la veuve Guérin, fileuse; la femme de Senard, ancien tessier et actuellement mendiant, demeurante à la rangée du cimetière des pauvres; la femme de Lemoine, ci-devant tessier, et actuellement paraletique; la veuve Lelait, journalière buandière, demeurante à la rangée du cimetière des pauvres ${ }^{55}$."

\section{Déclin des charités testamentaires}

Quelle est l'importance de toutes ces charités testamentaires et est-il possible de discerner une évolution?

La mesure en est impossible en raison de la diversité des dons, de l'absence de valeur vénale pour les legs en grains et en vêtements, de la liberté fréquemment laissée à l'exécuteur testamentaire de faire à sa convenance les legs aux pauvres, enfin de l'absence de listes de testaments. En effet, ainsi qu'il a été expliqué dans l'article précédemment évoqué ${ }^{56}$, la médiocrité de l'enregistrement dans le contrôle des actes a conduit à utiliser les minutes notariales, cependant il y a de nombreuses lacunes dans les collections de celles-ci et les répertoires des études rurales ont été rarement conservés.

Sans pouvoir être plus précis, on peut cependant dire que le nombre des testaments contenant des charités testamentaires diminue au cours du XVIII ${ }^{\mathrm{e}}$ siècle. Sur l'échantillon de 9000 testaments, on passe de 31,2\% durant la décennie 1700 à 1709 à $21,1 \%$ en 1780-1789. Ce déclin des charités testamentaires est renforcé par une diminution du nombre des testaments pieux, ainsi sur la paroisse de la Trinité d'Angers, y a-t-il 363 testaments pour 1250 décès de majeurs entre 1715 et 1724 , soit un taux de $29 \%$, et 169 testaments pour 1250 décès entre 1784 et 1793, soit 14 \%. Les conséquences sont graves pour les pauvres, puisque l'aide repose en quasi-totalité sur la charité privée. En particulier, la situation financière des hôpitaux devient de plus en plus difficile ${ }^{57}$, aussi l'État est-il amené à intervenir dans la gestion et à mettre fin à une autonomie à laquelle les administrateurs étaient très attachés.

Est-il possible d'établir des différences entre les régions de l'Anjou? Existe-t-il des régions plus charitables les unes que les autres? Le principal apport de l'enquête dont les résultats ont été précédemment publiés a été de montrer l'existence de fortes disparités dans la pratique testamentaire entre les régions de la province. Elle décline très tôt dans le Baugeois où les premiers indices se font sentir dès 1730 , et le mouvement s'accélère à partir de 1740; il en est de même dans la ville de Saumur (37 \% de clercs exécuteurs testamentaires pour $53 \%$ de laïcs dès 1740 ) et de manière un

55. 26 août 1788, Arch. dép. de Maine-et-Loire, $5^{\mathrm{E}} 1 / 1131$.

56. $A B P O$, t. 116, 2009 (2), p. 107.

57. IMBERT, Jean, Histoire des hôpitaux en France, Toulouse, 1982, p. 173-174 et 239 
peu plus lente dans celle d'Angers. La région des Mauges offre un tableau entièrement différent : certes il y a moins de testaments pieux, mais le déclin est beaucoup plus lent. Ici, les clercs exécuteurs testamentaires sont toujours majoritaires; le pourcentage des demandes de messes " pour le salut de l'âme " demeure le plus élevé de tout l'Anjou, avec 84 messes en moyenne par testament de 1700 à 1740, 51 de 1740 à 1760, 50 après 1760 . Les autres régions de la province se situent entre ces deux extrêmes : dans le Segréen, le mouvement de rupture se fait sentir à partir de 1750; dans la région entre Mayenne et Loir vers 1770, donc plus tard encore; le comportement des habitants des campagnes du Saumurois est proche de celui des fidèles des Mauges.

Pour les charités testamentaires, les résultats sont un peu différents. La première place revient au nord-ouest de la province avec $34,5 \%$ de testaments charitables dans la région située entre Mayenne et Loir et 33,5\% dans le Segréen; elles sont suivies par la ville de Saumur (24,5\%), puis le Baugeois (23,5 \%) et la ville d'Angers (22,8 \%). Dans les campagnes du Saumurois (22\%) et dans celles de la " quinte " d'Angers (20,8 \%), les testateurs sont un peu moins généreux. Le paradoxe est que la région des Mauges est à la dernière position (15\%), alors qu'elle donne, pendant tout le $\mathrm{XVIII}^{\mathrm{e}}$ siècle, des marques de piété; c'est que beaucoup de testaments contiennent la mention : "à l'égard des autres prières et aumônes, il (elle) s'en rapporte à son exécuteur testamentaire ", et celui-ci, dans la majorité des actes, est un clerc.

Au-delà de ces disparités régionales, le plus important sans aucun doute est la diminution de près d'un tiers du nombre des charités testamentaires, ce qui a pour conséquence des difficultés financières croissantes, tant pour les hôpitaux que pour les pauvres en général. Pour remédier à ce déficit l'État est amené à intervenir. Ainsi à la charité, manifestation du don avec un " contre-don » de valeur inestimable puisqu'il s'agit de la béatitude éternelle, succède l'assistance publique. 


\section{RÉSUMÉ}

Dans un corpus de plus de 9000 testaments, le quart des actes environ comporte des dons de grains, de vêtements ou d'argent, qui répondent aux prescriptions de l'Évangile, rappelées par les clercs. Les donateurs, dont les plus nombreux sont des gens fortunés, nobles, officiers, ecclésiastiques, professions " libérales ", marchands, attendent en retour des prières qui leur faciliteront l'accès au Paradis. Ils établissent donc une relation entre la distribution des dons et les services pour le repos de l'âme du défunt. Le nombre de telles clauses diminue d'un tiers au cours du XVIII ${ }^{\mathrm{e}}$ siècle, aussi les pauvres et les institutions chargées de les accueillir, tels les hôpitaux, ont des difficultés croissantes. L'État est amené à intervenir pour les régler, et l'assistance remplace la charité.

\section{ABSTRACT}

Within a corpus of over 9.000 testaments, about a quarter of the acts contains gifts of grains, clothes or money, according to the prescriptions of the gospel taught by the clergy. The donors, most of whom are wealthy people, nobles, officers, clergymen, members of the professions, merchants, expect in return prayers that will make it easier for them to go to Paradise. They therefore establish a connection between the distribution of gifts and the celebration of services for the soul of the deceased. The number of such clauses goes down by a third in the course of the $18^{\text {th }}$ century; consequently, the paupers and the institutions taking care of them, such as hospitals, face increasing difficulties. The state is led to intervene, and public welfare replaces private charity. 



\title{
Une phalange républicaine dans l'arrondissement de Saint-Malo : les Bleus de Bretagne (1902-1914)
}

\author{
Loïc THOMAS \\ Professeur certifié de Lettres (ER)
}

\begin{abstract}
L'Association des Bleus de Bretagne, fondée à Paris en 1899, rassemble des républicains laïcs et anticléricaux, d'abord dans la capitale, puis en Bretagne où les groupements locaux connaissent des succès très relatifs. La section de Saint-Malo se distinguera par son importance, son organisation ${ }^{1}$ et son dynamisme, au point qu'elle sera donnée en exemple aux autres sections ${ }^{2}$.

Ces militants de l'idée laïque vont animer l'arrondissement de Saint-Malo avec méthode et vigueur, s'installer à la tête de nombreuses mairies (dont celle de Saint-Malo), entrer au conseil général et gagner, avant le premier conflit mondial, les deux circonscriptions de l'arrondissement. Leur succès politique sera total et cette réussite entraînera l'anéantissement du parti monarchiste libéral catholique qui avait tenu les deux circonscriptions du pays malouin entre 1889 et 1893. Les représentants de ce parti, CharlesEmile La Chambre et le vicomte de Lorgeril, seront remplacés par les républicains François Brune et Léonce Demalvilain (auquel succèdera Alphonse Hervoches après la démission de Demalvilain en 1897) mais, au début du nouveau siècle, l'engagement de ces hommes en faveur de la République sociale fut jugé insuffisant par des membres des comités locaux dont certains accueillirent avec satisfaction l'idée de s'associer à la jeune ligue parisienne des Bleus de Bretagne. Leur adhésion à cette association républicaine va alors donner aux militants républicains, (dont plusieurs sont affiliés à la francmaçonnerie) un élan et une direction en tout point remarquables.
\end{abstract}

Sous l'impulsion de l'agent voyer Charles Fouré (1854-1931), une organisation politique d'encadrement et des réalisations notoires vont surgir

1. Le Beguec, Gilles, L'Entrée au Palis-Bourbon. Les filières privilégiées d'accès à la fonction parlementaire. 1919-1939. Thèse d'État. Université de Paris X Nanterre. p. 1199.

2. La Bretagne nouvelle. Juillet 1906. Discours du Président Armand Dayot. p. 25. 